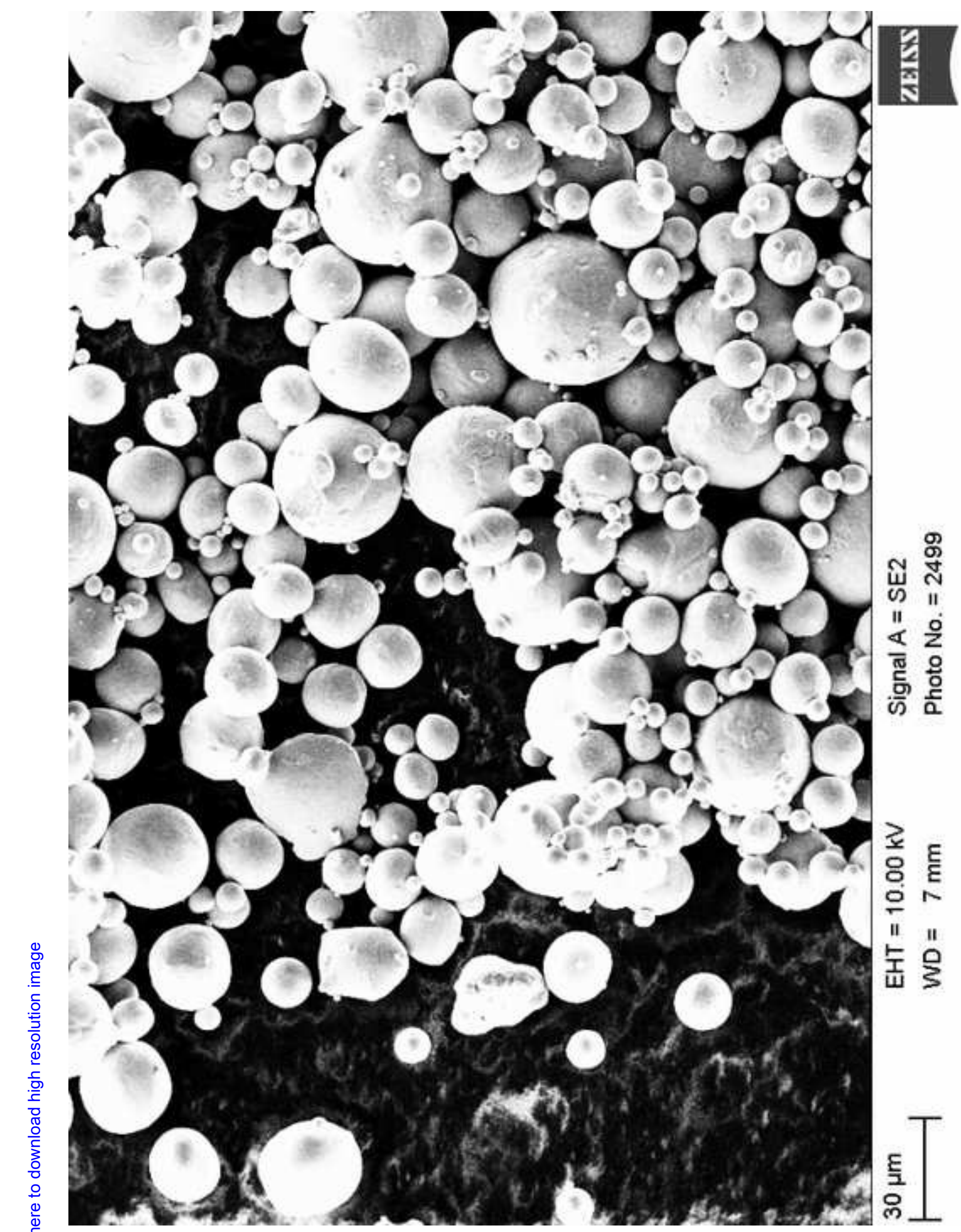




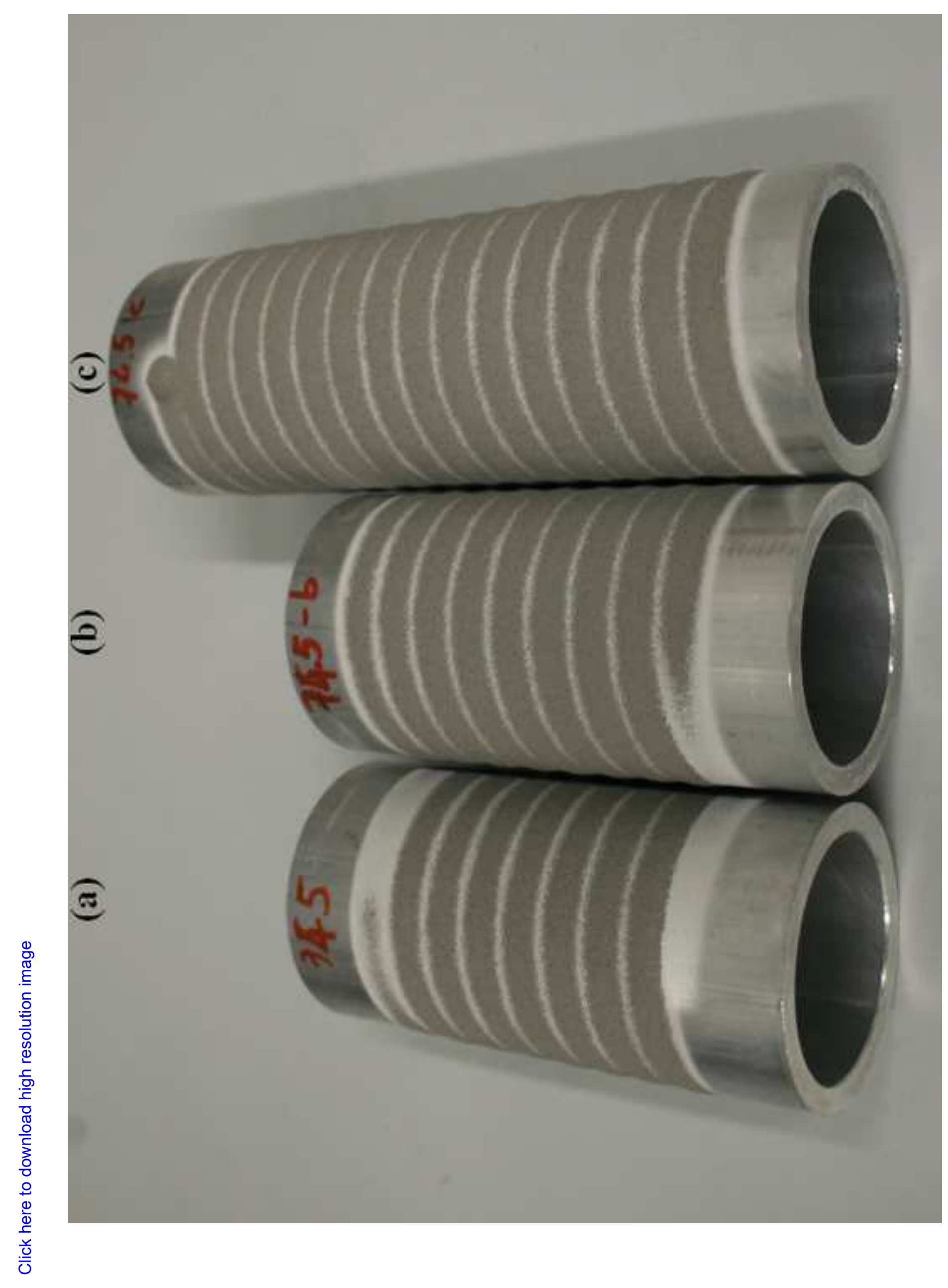




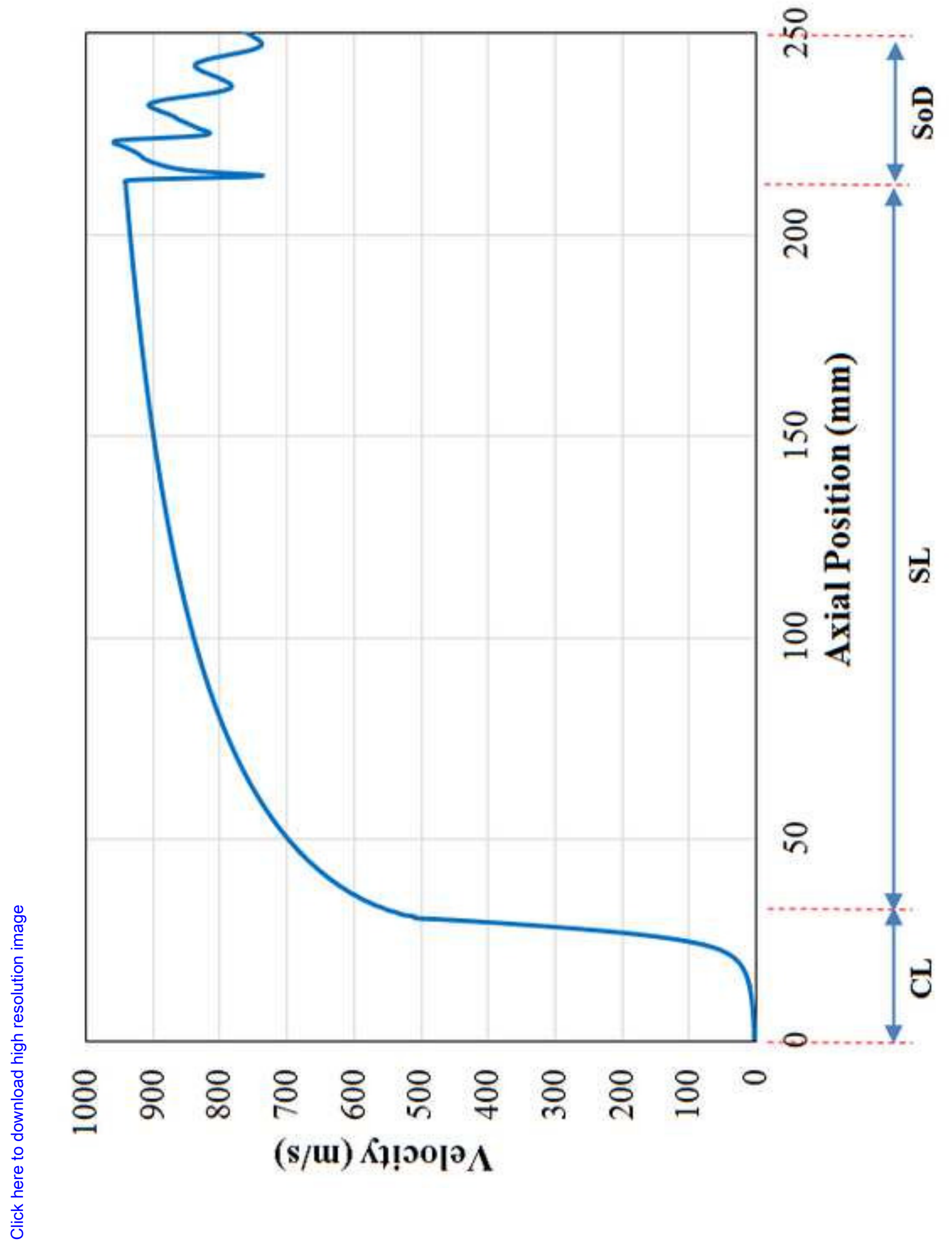




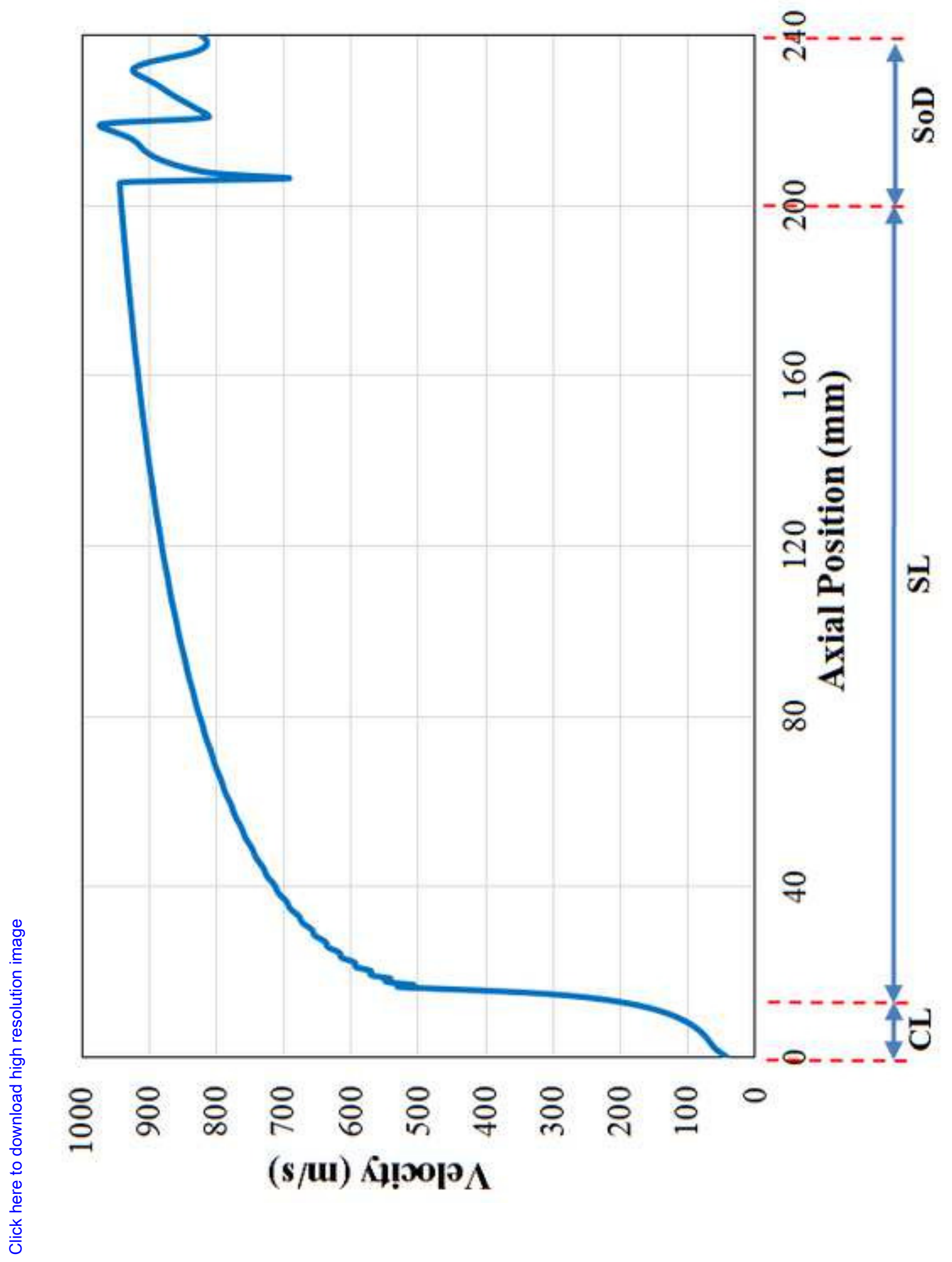




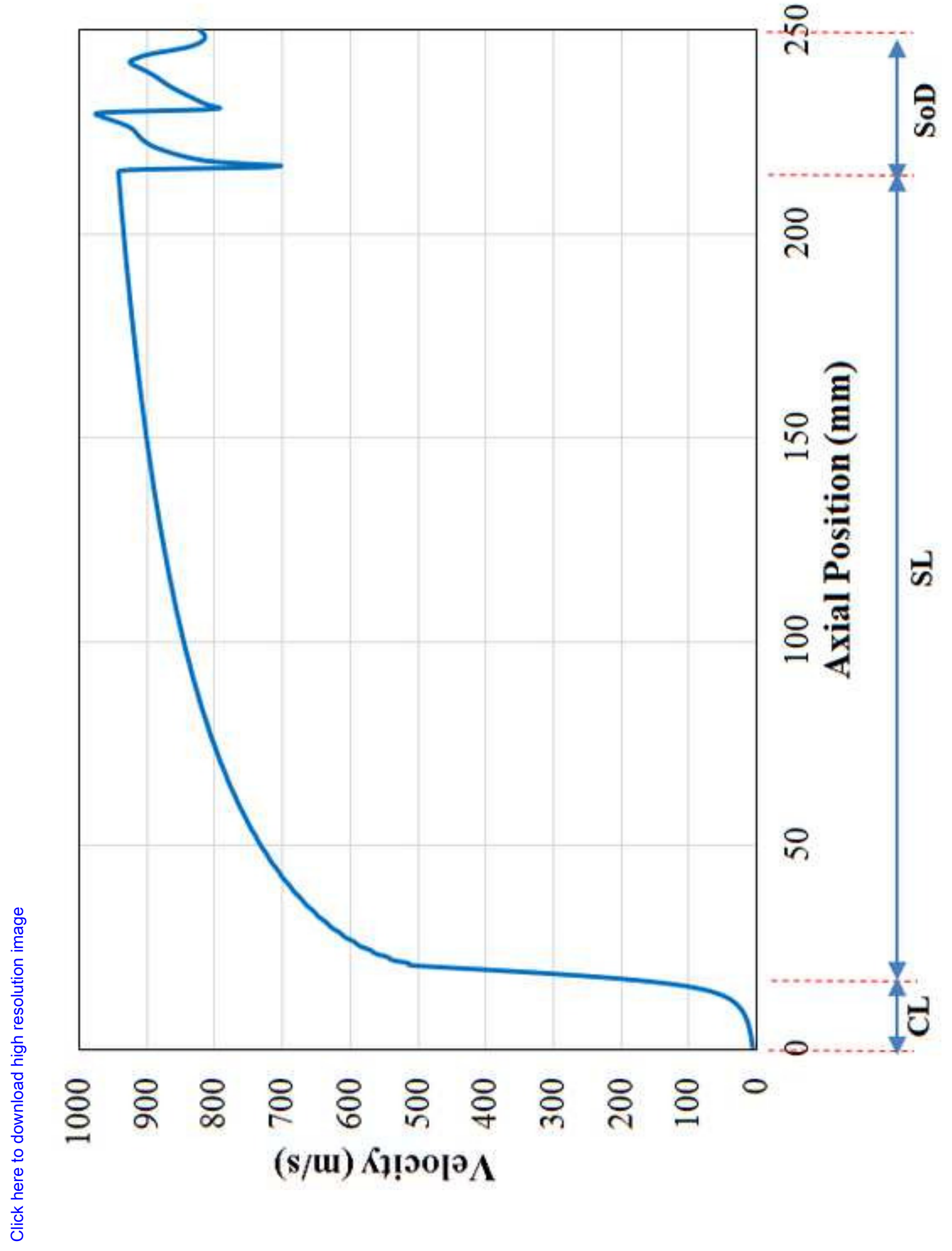




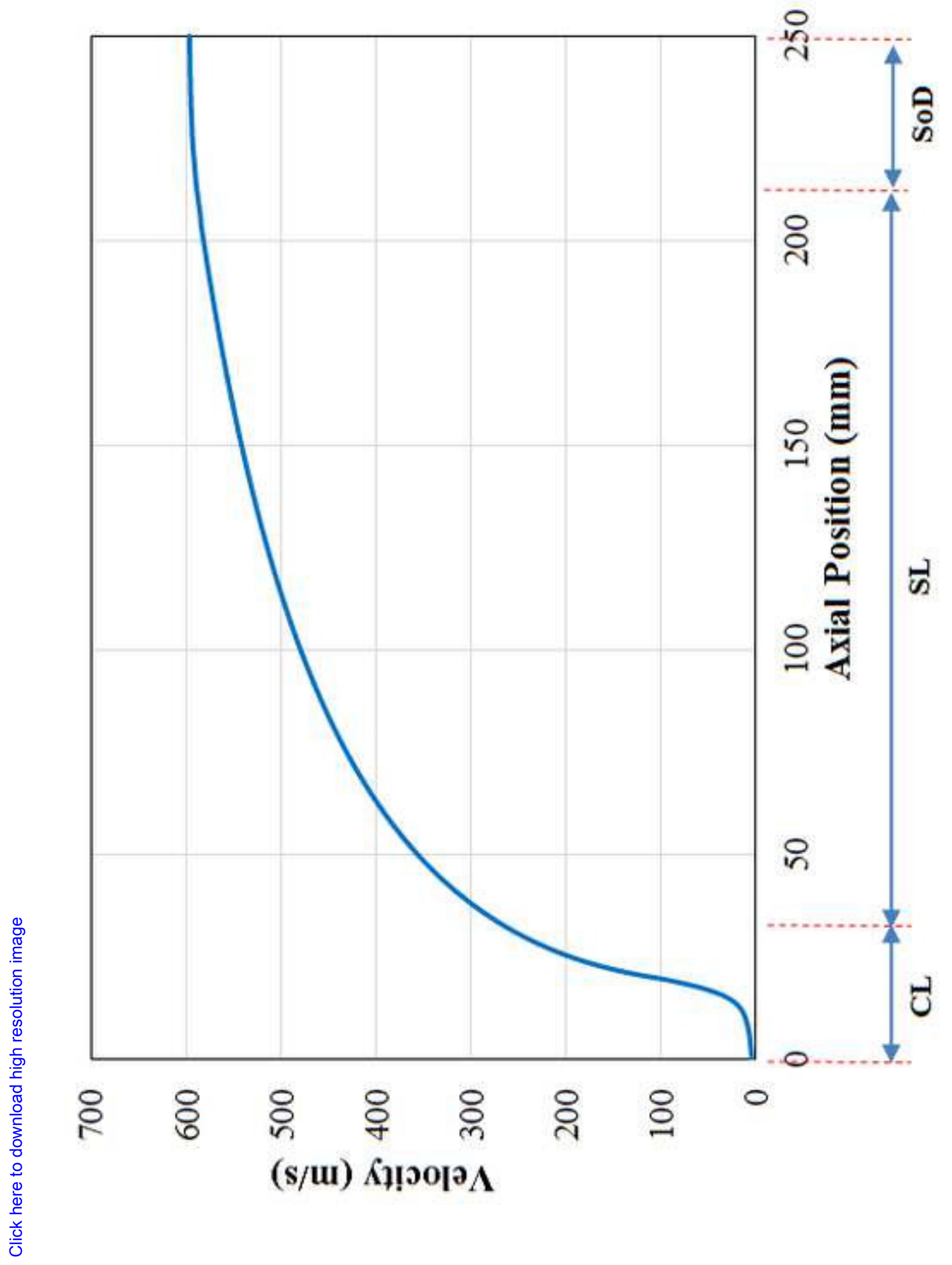




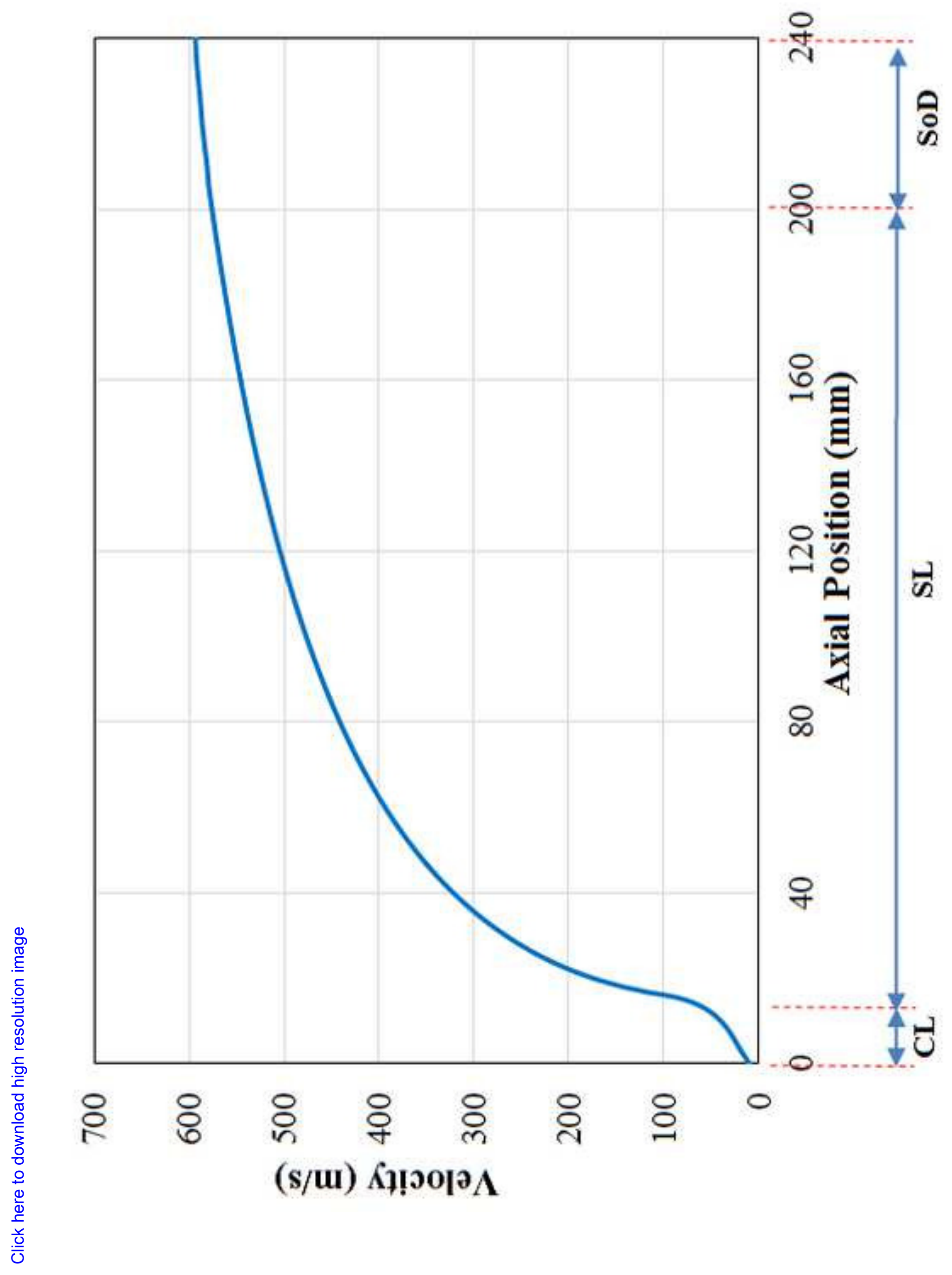




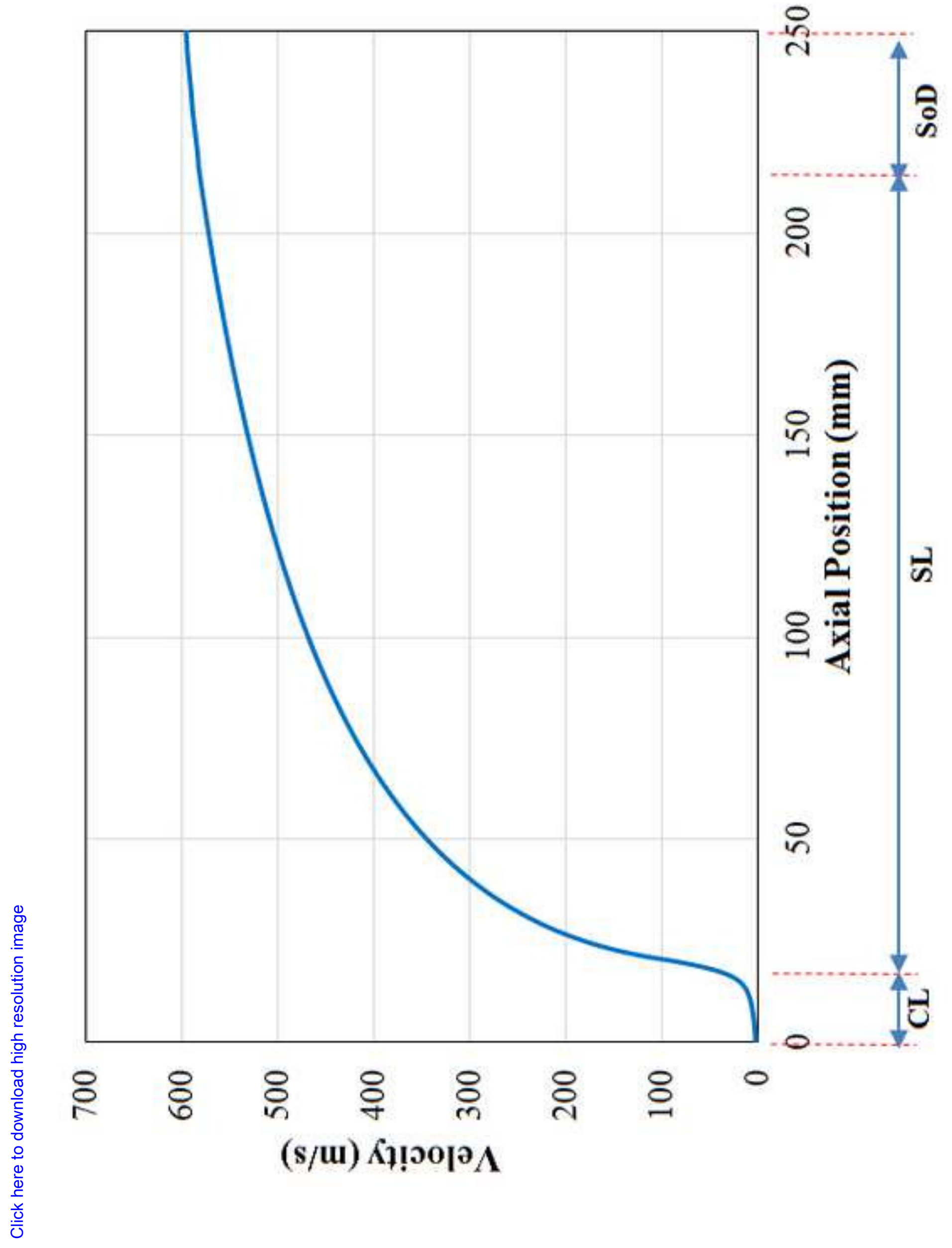




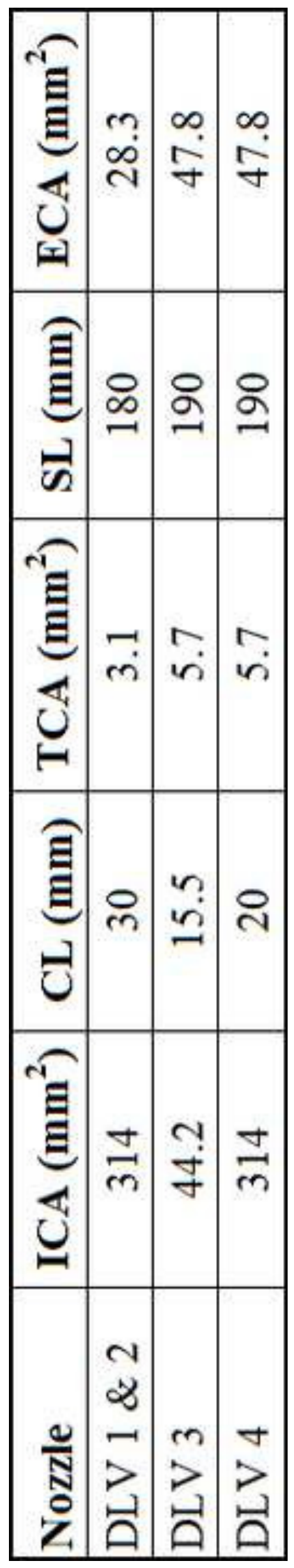




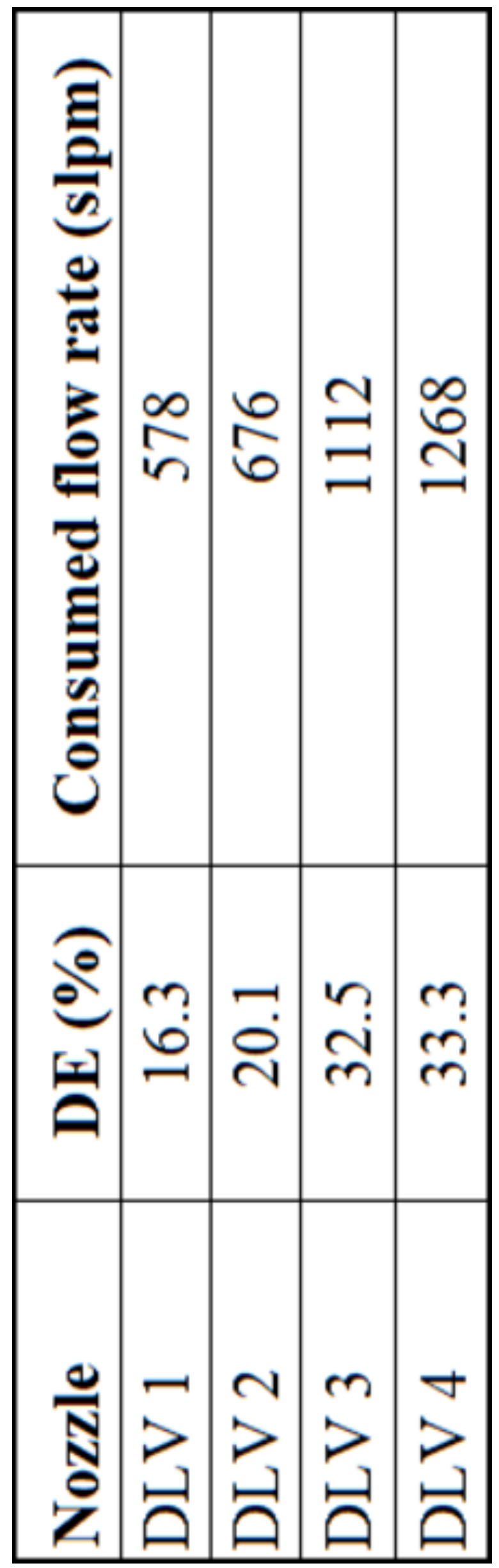




\title{
Current Design and Performance of Cold Spray Nozzles: Experimental and Numerical Observations on Deposition Efficiency and Particle Velocity
}

\author{
R. Lupoi \\ Trinity College Dublin, Department of Mechanical \& Manufacturing Engineering, \\ Parsons Building, Dublin 2, Ireland. \\ E-mail: LUPOIR@tcd.ie Secondary E-mail: $\underline{\text { rlupoi@yahoo.co.uk }}$ \\ Phone: $+35318961729 \quad$ Fax: +35316795554
}

\begin{abstract}
Coating technologies play a critical role in the world-wide manufacturing industry. The ability to form layers of specific materials onto engineering components to enhance mechanical and physical properties have numerous applications, ranging from corrosion protection, repair, hardfacing, down to purely aesthetic purposes. Cold Spray is an innovative technology, which allows for the manufacturing of layers in a solid-state manner, hence feedstock properties can be fully preserved. Its working principles relies upon the acceleration of powders up to supersonic velocities, and the subsequent generation of high energy impacts on a substrate which triggers the coating formation. This paper presents Deposition Efficiency (DE) results from four different supersonic nozzles when using titanium as feedstock material. DE is the most critical parameter to assess the performance of CS nozzles. A theoretical analysis through Computational Fluid Dynamics (CFD) is carried out so to compare numerical results against experimental findings. Results have suggested that current commercial codes cannot accurately predict the acceleration process under realistic working conditions. It is therefore difficult to predict DE levels. CS is starting to be applied to high-end engineering, however it is likely not to be successful unless
\end{abstract}


critical understanding is generated and used to accurately design nozzles, and predict the performance of new prototypes.

Key-words: Cold Spray, Titanium Coatings, CFD, Particle Velocity, Deposition Efficiency, Nozzle Design.

\section{Introduction}

The exponential raise for higher quality components combined with the need of reducing productions costs is currently exposing many conventional technologies as obsolete and not capable of delivering up to new required standards. In this respect, there is a strong push from the industrial community for the development and establishment of the new generation manufacturing processes, which will eventually provide a technological step-change towards product quality, production rates, costs reduction and environmental compatibility.

An important area is related to the capability of producing coatings. Coatings can be applied onto engineering components to locally improve their mechanical and physical characteristics, and represents in many cases the most efficient and economical solution in order to achieve the necessary product properties. In order to achieve the bulk deposition of layers of metals onto other types of metals or alloys, a number of techniques are currently available. The most common are Laser Cladding (LC) [1-7], Flame Spray (FS) [8-9], Plasma Spray (PS) [10-11], Detonation Gun (D-gun) [12] and High Velocity Oxy-Fuel (HVOF) [13-14]. All these methods are based upon the melting or partial melting of the substrate and coating material, with a number of serious disadvantages. As an example, the HVOF process was considered unfeasible for the deposition of titanium on large sea pillars for corrosion protection due to the high level of oxygen and oxides entrained in the deposits and the consequent detrimental properties [15]. 
Advanced materials, such as WC-Co, are increasingly attracting the industrial interest due to their excellent corrosion and wear resistance properties combined with environmental compatibility; it is however not possible to process them with conventional thermal spray techniques without inducing a level of decarburization [16].

Another technology, known as Cold Spray (CS), is based upon different a working mechanisms [17-18]. It has the potential to offer superior coating characteristics and to resolve the major weaknesses of the vast majority of methods. High pressure gas (nitrogen or helium) is accelerated in a converging-diverging De-Laval supersonic nozzle, reaching velocities well in excess of $1000 \mathrm{~m} / \mathrm{s}$. The feedstock material (in the form of powder) is fed through a feeder in the inlet zone of the nozzle; particles are dragged by the fast expanding main (or carrier) gas and reach high speed levels. When striking against a substrate occurs, particles plastically deform and bond to form a coating. This technology was proven to be completely solid-state (free of melting) [18-21], and has been successfully applied for the deposition of a wide variety of materials including Ti and its alloys, WC-Co, Super-alloys (In625) and others [22-25] typically onto metal components. Coatings can exhibit good properties, high bond strength with the substrate, low porosity (high density), and are completely oxide-free. Very interesting results have also shown the capability to process combinations such as Hydroxyapatite (HAP) mixed with titanium powders efficiently and at low cost, for the deposition of compatible coatings on biomedical implants to improve the bond strength with bone cells [26], and the possibility to add a laser to the system in order manufacture Stellite-6 coatings with nitrogen as carrier gas and without the necessity of crossing melting temperatures [27].

In a CS system, the most important component is the supersonic nozzle, and its capability to generate high Deposition Efficiency (DE) levels is critical when considering potential industrial 
applications. Within the state of the art, several publications specifically discuss the design of nozzles under different aspects. Recently, Sova et al. [28] have introduced CS "micro-nozzles", in the attempt to reduce the particle beam width to allow for the manufacturing of narrow deposits. In [29] the authors approach a similar problem, however they propose a different nozzle solution developed through an analysis of particle dispersion in the acceleration process. Other authors such as in [30] and [31] have on the other hand concentrated their studies on the particle acceleration process through numerical investigations, with the aim of generating insights on trajectories and velocity distributions for standard nozzle configurations.

However, there is certainty a lack of studies where different nozzle geometries are compared (experimentally and theoretically) against their DE performance and results are accurately explained. It is well known that in order to achieve a high DE, impact particle velocities must be well above the critical or minimum level to achieve deposition. Thus, at the design stage of the nozzle, it is practical to study the particle acceleration process to obtain information on achievable speeds, so to have an estimation of potential DEs. Within the state of the art, "Lagrangian" or "one-way coupling" techniques are common theoretical approaches to solve the particle velocity, however it is typically assumed that carrier gas phase and particles interaction are negligible. The work presented in this paper discuss the DE performance of four different nozzle designs for the deposition of titanium powder over aluminium tubes, when operating conditions are maintained constant. The paper also provides a first attempt to explain the behaviour of each nozzle, and how such could be in fact directly related to the consumed flow rate and carrier gas-particle interactions. 


\section{Experimentation}

\subsection{CS System}

The CS apparatus (nitrogen type) installed within the University of Cambridge (UK) premises was used for the development of this work. The system comprises of an open-loop high pressure powder feeder (Praxair 1264-HP), a gas-heater (CGT Kinetics 3000) and a computerized nitrogen handling system allowing for a maximum working pressure of $3 \mathrm{MPa}$. The powder feeder includes a load cell, so to allow for the measurement of powder mass flow rate during processing. A flow meter is also in place to read the consumed flow rate during processing of both powder feeder and main (carrier) gas lines. Nitrogen through the main line is fed into the gas-heater from comemrcial Manifold Cylinder Pallets (MCPs) and preheated prior entering the nozzle, for a maximum allowable inlet temperature of $500{ }^{0} \mathrm{C}$. It is well understood that a higher gas temperature will generate improved velocities at the nozzle exit; i.e. particle speed. The system was designed in such a way to easily interchange the type of supersonic nozzles used, each of them designed to operate under a specific set of processing conditions and powder materials. I order to produce coatings onto surfaces, a CNC X-Y table is installed and it is used to move the substrate as required; hence the nozzle is kept static and on a vertical position during processing. A motorized spindle (fitted on the upper surface of the X-Y table) was also included in the system to allow for the manufacturing of coatings onto cylindrical components.

\section{$\underline{2.2 \text { Spray Results }}$}

Titanium powder (CP-grade 2, $-45 \mu \mathrm{m}$ size, spherical geometry, purchased from Active Metals Ltd., UK) was used as feedstock material. Figure 1 shows a SEM picture of the feedstock as received from the factory, it is clear the particulate spherical geometry can be therefore confirmed. The selected substrate material was in the form of tubes, made out of an aluminium 
alloy (6082-T6). The tubes measure $50 \mathrm{~mm}$ in external diameter, with a wall thickness of approximately $6 \mathrm{~mm}$, and were not subjected to any treatment prior being coated.

The aim of this work was to assess the performance of different nozzle geometries, primarily against $\mathrm{DE}$. In this respect, a set of experiments was designed where the processing conditions were kept constant, so to easily compare the performance of the nozzles used. A total of four nozzles were experimented, relevant geometrical details are summarized in Table 1. The nozzles, namely DLV 1, 2, 3 and 4 are characterized by an internal converging-diverging profile with a circular cross-section to resemble a conventional De-Laval type of geometry. DLV 1 and 2 are made from the same in-house design, they are in fact theoretically identical. They have been however manufactured from different providers and by using dissimilar techniques. On the other hand, DLV 3 is a commercial nozzle, while DLV 4 is another in-house design. With respect to Table 1, ICA represents the inlet cross-sectional area, $\mathrm{CL}$ is the converging section length, TCA is the throat or restriction cross-sectional area, SL is the length of the supersonic section and ECA is the exit cross-sectional area. The dimensions reported in the table are nominal, hence a tolerance of $\pm 25 \mu \mathrm{m}$ must be applied. In all cases, the nozzles were made in WC-Co to minimize the particles abrasion effect on the nozzle wall due to the excellent wear resistant properties of this material. The internal profile surface finish was kept below $0.5 \mu \mathrm{m}$ Ra, while all designs were manufactured by following a "monolithic" strategy, i.e. in one piece only.

The processing conditions were kept constant when testing each of the arrangements. The nozzle inlet pressure was adjusted to $3 \mathrm{MPa}$, while the nitrogen inlet temperature was set to $350{ }^{\circ} \mathrm{C}$. This temperature level is not expected to produce the required exit velocities to achieve high $\mathrm{DE}$ conditions with titanium powder, however such is not the purpose of this work. As the system is required to cool down after processing to allow for a nozzle interchange, the use of higher 
temperatures would have considerably increased the experimental lead times and made no difference in final results with respect to nozzles relative comparison. The tubes were mounted in the spindle, and a set rotational velocity was imposed to generate a linear Transverse Speed (TS) of $50 \mathrm{~mm} / \mathrm{s}$ at correspondence of the outer diameter. In all experiments the Standoff Distance (SoD) between the nozzle exit and the substrate material was $40 \mathrm{~mm}$. The powder feeder wheel speed was adjusted to approximately half of the achievable maximum. In all configurations a mixture of powder and gas (nitrogen) from the feeder was injected in the nozzle inlet, therefore within the subsonic region.

Figure 2 shows three examples of manufactured titanium coatings, with the DLV 4 configuration. The CNC table was programmed to translate at a set speed while the spindle rotates, in such a way to produce a "spiral", i.e. a continuous track. As the powder mass flow rate was measured through the powder feeder load cell, the processing time had to be long enough for the load cell to record a perceivable loss in weight rate. In this respect, shorter and longer runs were executed in order to estimate a critical run time. The tubes were weighed prior and after being processed; it was therefore possible to calculate the DE level. Figure 2(a) shows a coated tube for a total run time of 26sec. In Figure 2(b) the sample as in Figure 2(a) was reproduced to confirm the measured DE, while Figure 2(c) shows a coating obtained with a longer experiment (54sec.) to ensure no significant changes in DE were acknowledged against the shorter runs.

Relevant experimental results by the four nozzle configurations are summarized in Table 2 . The table reports the calculated DE, but also includes the consumed nitrogen flow rate by each individual set up (it has been reported as it will provide argument for discussion in the sections to follow). The powder feed rate was slightly different in each trial despite the wheel speed was 
maintained constant, and measured within the range of $55 \pm 9 \mathrm{~g} / \mathrm{min}$. This behaviour is rather typical for the type of powder feeder used, being an open-loop system.

It is not challenging to understand the reason of why each set up is consuming a different level of nitrogen, being this parameter directly related to TCA. As the pressure and temperature at the inlet were maintained constant $\left(3 \mathrm{MPa}, 350^{\circ} \mathrm{C}\right)$, a nozzle with a larger TCA would require a higher flow in order to generate the working conditions. DLV 1 and DLV 2, although being from the same nominal design, are in fact consuming a slightly different level of gas due to manufacturing tolerances related effects; the TCA of DLV 2 is actually larger. DLV 3 and DLV 4 are different in overall design, but have the same nominal TCA; this is the reason of why they both consume a similar flow.

On the other hand, a harder task is to understand why the DE is different. It dramatically increases from $16.3 \%$ with DLV 1 up to $33.3 \%$ with DLV 4. For the specific case of these experiments $\mathrm{DE}$ can be assumed to be directly related to the maximum achievable particle velocity; the particle and carrier gas acceleration process is therefore studied and presented in the sections to follow.

\section{Nozzles Performance Prediction}

\subsection{Simulation Set up}

The four configurations as by Table 1 were simulated under a set level of operating conditions, using Computational Fluid Dynamics (CFD) through Ansys-Fluent v14.0. Nitrogen was used as operating fluid, and the ideal gas law was employed to take into account variations of density. The density based solver was used under steady-state conditions, as it better complies with compressible flows at supersonic regimes. It was possible in all cases to achieve a grid- 
independent solution with a maximum grid elements number of approximately 120000 (the nozzles nominal dimensions were used within the Ansys-Workbench environment to build the geometrical models). The nozzles are all of circular cross-section, therefore the models were developed in 2D; however the governing equations in axial-symmetric form were solved. This emulates 3D effects when axial-symmetry applies. The simulations were solved up to the second order discretization, with the necessary number of iterations to achieve an acceptable level of convergence. A two equations $(\mathrm{k}-\varepsilon)$ turbulence model was used. The boundary conditions at the nozzle inlet (pressure inlet) were adjusted to $3 \mathrm{MPa}$ and $350^{\circ} \mathrm{C}$ in all cases, while atmospheric static pressure was set at the outlet zone of the model; however with the attention of constructing it far enough from the nozzles actual exit so not to impose unrealistic conditions at this location. Pattison et al. [32] have used Fluent with a similar simulation set-up to visualize the "bowshock" at the substrate interface in CS. CFD results have slightly overestimated experimental observations by a Schlieren analysis, but this tool was recognized valuable to predict carrier gas characteristics.

Stream of solid particles were released in the nozzles inlet zone, when using the Discrete Phase Modelling (DPM) algorithm and the "high-mach-number" drag law to compute particles acceleration. This procedure can simulate the acceleration of particulate flows of a given size and material in a carries gas, within a Lagrangian reference frame. This means that the effects of particles on the gas phase are not considered relevant. The vast majority of researchers in the field implement similar or very similar procedures to simulate the acceleration of particles in CS nozzles. 


\section{$\underline{3.2 \text { Carrier gas velocity }}$}

Figure 3 shows simulation results for DLV 1 and DLV 2. The gas velocity magnitude is represented across the entire nozzle length at correspondence of the axial location. The velocity is shown to rapidly increase from a nearly zero value, i.e. quasi-static conditions, at the beginning of CL up to a level between 900 and $1000 \mathrm{~m} / \mathrm{s}$ at the exit. The nozzle is over-expanded, as the carrier gas pressure at the exit is slightly lower than atmospheric. Designing a nozzle internal contour in this manner enables the generation of higher exit velocities. However, shockwaves forms and their effect on the velocity distribution after the exit cross-section is clearly visible within the $\mathrm{SoD}$ zone in the figure.

Figure 4 and Figure 5 shows the carrier gas velocity behaviour for DLV 3 and DLV 4 respectively. In DLV 3 low intensity shock-waves forms at correspondence of the nozzle restriction cross-section, i.e. at the end of CL, which are progressively dissipated as the carrier gas accelerates. This region is represented by a rather complex flow, as conditions evolve from sub-sonic to sonic and super-sonic within a very short distance; it is in fact difficult to completely avoid the formation of shock-waves through an appropriate nozzle contour design as flow conditions are typically not uniform within a cross-section. However, further CFD simulations have demonstrated that the addition of a fillet radius at the interception between CL and SL can make changes of flow properties more progressive, hence shock-waves can be minimized. As DLV 3 is a commercial design, it was not possible to take this into account.

All arrangements, although being characterized by a different internal profile, generate very similar accelerations and exit velocities. As reported in Table 2, the consumed nitrogen flow varies and experimental measurements are well in agreement with CFD results; however such has of course not resulted in any major difference in velocity distributions. Analytical approaches 
also confirm the theoretical independence of consumed flow rate with final Mach number and produced speed magnitude [33].

\subsection{Particle speed}

Figure 6 shows the particle velocity distribution in DLV 1 and DLV 2. A single titanium particle injection port was created in the nozzle inlet section (Axial Position $=0$ ) at the axis location, and using the top-end size of the commercial range $(45 \mu \mathrm{m})$ in the settings.

The particle accelerates, and it is predicted to reach a maximum velocity of $596 \mathrm{~m} / \mathrm{s}$ (approximately 63\% of the carrier gas speed) at the end of the SoD zone. It is interesting to notice the acceleration is still active after the nozzle exit, proving negligible interference by the carrier gas shock-waves in the $\mathrm{SoD}$ zone as by Figure 3. Injection ports were also created at various locations within the $\mathrm{CL}$ zone and not necessarily across the axis; however, very similar profile velocities were computed as by the one in Figure 6 demonstrating negligible differences. Figure 7 and Figure 8 reports the particle velocity distribution as by DLV 3 and DLV 4, where a maximum speed of $593 \mathrm{~m} / \mathrm{s}$ and $595 \mathrm{~m} / \mathrm{s}$ respectively is predicted.

It is also interesting to simulate the particle stream temperature as it moves in the nozzle and accelerates. As explained, powders (with no pre-heating) are injected in the inlet region where a gas temperature of $350^{\circ} \mathrm{C}$ is imposed. There is no doubt the particle temperature raises in this zone, however cooling during the acceleration process is predominant. In all cases, at the impact location it is not predicted to cross the $100^{\circ} \mathrm{C}$ level and it is therefore assumed not to play significant role to the coating formation of titanium and related DEs.

To summarize, CFD simulations results from Figure 3 to Figure 8 suggest that all of the arrangements, despite being different in design, are generating very similar performances for 
both carrier gas and particle. However, the experimental measurements in Table 2 appear to be in contradiction, as they are showing a dramatic difference in DE. As the nozzles operate exactly using the same processing conditions, powder and substrate materials, such behaviour is mainly explainable if the particle impact velocities are in reality different; presented results by the CFD analysis seem therefore inconclusive.

The work published by Samareh et al. [34] can be considered to provide an explanation. The authors have developed an initial but complex computational methodology to fully include particles interaction with the gas phase, by following an Eulerian-Eulerian approach. Aluminium was simulated as powder material injected at increasing rates in the carrier gas (nitrogen). The models had clearly shown a dramatic reduction in carrier gas velocity at the nozzle exit due to the interaction with particles, which was recognisable at feed rates as low as $6.69 \mathrm{~g} / \mathrm{min}$ in the simulations. Ultimately, particles would also find themselves travelling at considerably reduced levels, hence poor DEs can be explained.

The article by Samareh et al. does not report experimental measurements, but the authors overall explanation can be applied to the current work. It can be in fact considered suitable for an initial validation. Particle-gas interactions peak at correspondence of the TCA (see Table 1), being the smallest cross-section in the nozzle. This location is critical as the flow is changing its regime from sub-sonic to sonic and subsequently super-sonic. The presence of solid particles can negatively interfere with the progress of the transformation, and for higher loadings chocking conditions may arise. As TCA increases from DLV 1 to DLV 4 and the powder flow rate is maintained constant, the solid phase volume fraction reduces and interactions with the gas phase can be minimized. This phenomenon can certainly explain, at least at qualitative level, the differences in DE reported by Table 2. It is also true that with a larger TCA the carrier gas flow 
rate increases, and it is currently uncertain whether or not this parameter is also relevant to gasparticle interactions; the flow values have been however reported for completeness of data. The distributions from Figure 3 to Figure 8 can be therefore interpreted as the maximum theoretical particle and gas velocity each nozzle can generate, providing no internal interactions occur. It is of course reasonable to assume that feedstock materials can respond to this effect in dissimilar ways, and the losses of exit particle speed can, case by case, cause or not considerable differences in DE.

It can be concluded that particle to gas interactions are critical to estimate velocities and will affect DE. Current tools are not robust enough to provide an accurate prediction of its realistic effects through commercial CFD packages (nor how to overcome to them) for compressible flows at supersonic regimes. It will be vital in the short coming future to develop advanced numerical and theoretical models suitable for this process, with a higher reliability level. CS is expanding its applications range, however results from this article demonstrate that it is still not possible to accurately perform the more fundamental task of predicting the behaviour of particles in the nozzles. The CFD analysis presented in this article was in fact unable to differentiate each nozzle characteristic.

\section{Conclusions}

Coatings manufacturing is a very active research filed, where new technologies have been recently introduced and are potentially capable of replacing current methods. One of them is Cold Spray (CS), where feedstocks in the form of powder are fired upon a substrate material at supersonic speed through a carrier gas (nitrogen or helium). Coatings typically exhibit good properties, as this process is demonstrated to be fully free of melting. A critical component is the supersonic nozzle, and it is important to accurately predict DE levels through CFD studies to 
enable the assessing of performances at the design stage. Four nozzles were manufactured using different internal profiles, and were tested using the same processing conditions with titanium powder. Rather dissimilar levels of DE were measured on an aluminium alloy substrate (tube), ranging from $16.3 \%$ to $33.3 \%$. The nozzles were simulated by using CFD; however available theoretical predictions did not suggest any major difference in particle velocity distribution among the tested designs; the difference in achieved DE could not be directly explained. However, an initial solution to the problem concluded that solid-gas phase interactions (a phenomena very difficult to accurate model) can ultimately lead to a dramatic loss of particle speed at the nozzle exit, hence to a lower or higher DE. This is particularly true when higher particle loadings are fed in the nozzle. It is currently not possible to easily take this effect into account at supersonic regimes for compressible flows when using commercial software packages, and this is undoubtedly a strong limitation. More reliable tools are required to efficiently predict the performance of potential new nozzle prototypes.

\section{Acknowledgements}

The authors wish to express their gratitude to the Centre for Industrial Photonics (CIP) University of Cambridge (UK) research team for the valuable support in developing the experimental part of this work.

\section{References}

[1] E. Toyserkani, A. Khajepour, S.F. Corbin "Laser Cladding", CRC Press, 2004.

[2] M. Brandt, S. Sun, N. Alam, P. Bendeich, A. Bishop "Laser cladding repair of turbine blades in power plants: from research to commercialization", International Heat Treatment \& Surface Engineering, 2009, 3, 105-114.

[3] S. Sun, Y. Durandet, M. Brandt "Parametric investigation of pulsed Nd:YAG laser cladding of stellite-6 on stainless steel”, Surface \& Coating Technology, 2005, 194, 225-231. 
[4] K. Partes, G. Sepold, "Modulation of power density distribution in time and space for high speed laser cladding", Journal of Materials Processing Technology, 2008, 195, 27-33.

[5] R. Jendrzejewski, G. Sliwinski, M. Krawczuk, W. Ostachowicz "Temperature and stress fields induced during laser cladding”, Computers \& Structures, 2004, 82, 653-658.

[6] R. Jendrzejewski, G. Sliwinski, M. Krawczuk, and W. Ostachowicz "Temperature and stress during laser cladding of double-layer coatings", Surface \& Coatings Technology, 2006, 201, 3328-3334.

[7] G. Delette, J. Laurencin, S. Murer, D. Leguillon "Effect of residual stresses on the propagation of interface cracks between dissimilar brittle materials: Contribution of two and three-dimensional analyses", European Journal of Mechanics - A/Solids, 2012, 35, 97-110.

[8] G. Easter "Thermal Spraying - Plasma, ARC and Flame Spray Technology", Wexford College Press, 2008.

[9] J. A. Gan, C. C. Berndt, "Design and manufacture of Nd-Fe-B thick coatings by the thermal spray process”, Surface \& Coatings Technology, 201 1, 205, 4697-4704.

[10] L. Pawłowski "The science and engineering of thermal spray coatings", Wiley, 1995.

[11] Q. Wei, Z. Yin, H. Li "Oxidation control in plasma spraying NiCrCoAlY coating", Applied Surface Science, 2012, 258, 5094-5099.

[12] J. H. Kim, M. C. Kim, C. G. Park "Evaluation of functionally graded thermal barrier coatings fabricated by detonation gun spray technique", Surface \& Coatings Technology, 2003, $168,275-280$.

[13] V.V Sobolev, J. Guilemany, J.M. Guilemany "HVOF Spraying: Theory and Applications", The Institute of Materials, 1997.

[14] M. Li, P. D. Christofides "Computational study of particle in-flight behaviour in the HVOF thermal spray process", Chemical Engineering Science, 2006, 61, 6540-6552.

[15] S. Kuroda, M. Watanabe, K. Kim, H. Katanoda "Current status and future prospects on warm spray technology”, Journal of Thermal Spray Technology, 2011, 20, 653-676.

[16] C.-J. Li, G.-J. Yang "Relationships between feedstock structure, particle parameter, coating deposition, microstructure and properties for thermally sprayed conventional and nanostructured WC-Co", International Journal of Refractory Metals and Hard Materials, 2013, 39, 2-17.

[17] A.P. Alkhimov, A.N. Papyrin, V.F. Kosarev, N.I. Nesterovich, M.M. Shushpanov "GasDynamic Spraying Method for Applying a Coating", US5302414; WOWO9119016; EP0484533, year of priority (issued): 1990 (1994).

[18] V.K. Champagne "The Cold Spray Material Deposition Process: Fundamentals and Applications", Woodhead Publishing Ltd., 2007.

[19] T. Schmidt, F. Gartner, H. Assadi, H. Kreye "Development of a generalized parameter window for cold spray deposition", Acta Materialia, 2006, 54, 729-742.

[20] A. Moridi, S.M. H.-Gangaraj, M. Guagliano "A hybrid approach to determine critical and erosion velocities in the cold spray process", Applied Surface Science, 2013, 273, 617-624. 
[21] D. Goldbaum, J. M. Shockley, R. R. Chromik, A. Rezaeian, S. Yue, J.-G. Legoux, E. Irissou "The Effect of Deposition Conditions on Adhesion Strength of Ti and Ti6Al4V Cold Spray Splats", Journal of Thermal Spray Technology, 2012, 21(2), 288-303.

[22] T. Marrocco, T. Hussain, D.G. McCartney, P.H. Shipway "Corrosion Performance of Laser Post-treated Cold Sprayed Titanium Coatings", Journal of Thermal Spray Technology, 2011, 24(4), 909-917.

[23] A. S. M. Ang, C. C. Berndt, P. Cheang "Deposition effects of WC particle size on cold sprayed WC-Co coatings", Surface \& Coatings Technology, 2011, 205, 3260-3267.

[24] P.-H. Gao, C.-J. Li, G.-J. Yang, Yi-G. Li, C.-X. Li "Influence of substrate hardness transition on built-up of nanostructured WC-12Co by cold spraying", Applied Surface Science, 2010, 256, 2263-2268.

[25] P. Poza, C.J. Múnez, M.A.G.-Maneiro, S. Vezzu, S. Rech, A. Trentin "Mechanical properties of Inconel 625 cold-sprayed coatings after laser remelting. Depth sensing indentation analysis", Surface \& Coating Technology, 2012, Article in Press.

[26] X. Zhou, P. Mohanty "Electrochemical behavior of cold sprayed hydroxyapatite/titanium composite in Hanks solution", Electrochimica Acta, 2012, 65, 134-140.

[27] R. Lupoi, A.Cockburn, C. Bryan, M. Sparkes, F. Luo, W.O'Neill "Hardfacing steel with nanostructured coatings of Stellite-6 by supersonic laser deposition", Light: Science and Applications (Nature Publishing Group), 2012, 1, 1-6.

[28] A. Sova, A. Okunkova, S. Grigoriev, I. Smurov "Velocity of the particles accelerated by a cold spray micronozzle: Experimental measurements and numerical simulation", Journal of Thermal Spray Technology, 2013, 22(1), 75-80.

[29] R. Lupoi, W. O’Neill "Powder stream characteristics in cold spray nozzles", Surface \& Coatings Technology, 2011, 206, 1069-1076.

[30] H. Tabbara, S. Gu, D.G. McCartney, T.S. Price, P.H. Shipway "Study on process optimization of cold gas spraying", Journal of Thermal Spray Technology, 2011, 20(3), 608-620.

[31] S.Li, B. Muddle, M. Jahedi, J. Soria "A numerical investigation of the cold spray process using underexpanded and overexpanded jets", Journal of Thermal Spray Technology, 2011, 21(1), 108-120.

[32] J. Pattison, S.Celotto, A.Khan, W.O'Neill "Standoff distance and bow shock phenomena in the Cold Spray process", Surface \& Coatings Technology, 2008, 202, 1443-1454.

[33] R.C. Dykhuizen, M.F. Smith "Gas dynamic principles of Cold Spray", Journal of Thermal Spray Technology, 1998, 7(2), 205-212.

[34] B. Samareh, A. Dolatabaldi "Dense particulate flow in a cold gas dynamic spray system", Journal of Fluids Engineering - Transaction of the ASME, 2008, 130(8). 


\section{List of Figure Captions}

Figure 1: SEM picture of the feedstock material (Ti CP-grade2, $-45 \mu \mathrm{m}$ size).

Figure 2: (a) Titanium coatings (single pass) onto Aluminium 6082-T6 tubes measuring 50mm in outer diameter with DLV 4 configuration. (b) Repetition. (c) Longer run.

Figure 3: Velocity magnitude distribution by a CFD analysis for DLV 1 and DLV 2.

Figure 4: Velocity magnitude distribution by a CFD analysis for DLV 3.

Figure 5: Velocity magnitude distribution by a CFD analysis for DLV 4.

Figure 6: Particle velocity magnitude distribution by a CFD analysis for DLV 1 and DLV 2.

Figure 7: Particle velocity magnitude distribution by a CFD analysis for DLV 3.

Figure 8: Particle velocity magnitude distribution by a CFD analysis for DLV 4.

\section{List of Tables}

Table 1: Nozzles relevant geometrical details.

Table 2: Experimental results of Deposition Efficiency and nitrogen flow consumed by each nozzle $\left(3 \mathrm{MPa}, 350^{\circ} \mathrm{C}\right)$. 